\title{
Continuous insulin infusion reduces mortality in patients with diabetes undergoing coronary artery bypass grafting
}

\author{
Anthony P. Furnary, MD, a,d Guangqiang Gao, MD, ${ }^{a}$ Gary L. Grunkemeier, PhD, ${ }^{\mathrm{b}}$ YingXing Wu, MD, ${ }^{\mathrm{b}}$
} Kathryn J. Zerr, MBA, ${ }^{b}$ Stephen O. Bookin, MD, ${ }^{c}$ H. Storm Floten, MD, ${ }^{\text {add }}$ and Albert Starr, MD ${ }^{a, d}$

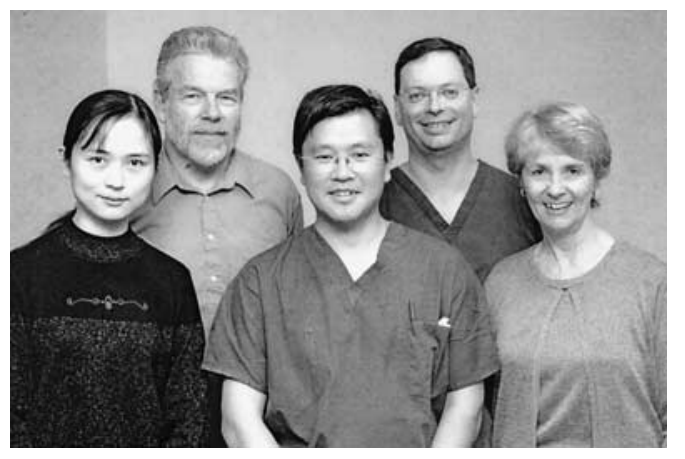

Left to right: Grunkemeier, Furnary (back row); $\mathrm{Wu}, \mathrm{Gao}$, Zerr (front row)

See related editorial on page 985.

From the Department of Cardiothoracic Surgery, Providence St Vincent Medical Center, ${ }^{\text {a }}$ the Medical Data Research Center, Providence Health Systems, ${ }^{\text {b }}$ the Department of Endocrinology, ${ }^{\mathrm{c}}$ and the Department of Surgery, ${ }^{\mathrm{d}}$ Oregon Health and Science University, Portland, Ore.

Read at the Eighty-second Annual Meeting of The American Association for Thoracic Surgery, Washington, DC, May 5-8, 2002.

Received for publication May 15, 2002; revisions requested July 22, 2002; revisions received Aug 23, 2002; accepted for publication Sept 30, 2002.

Address for reprints: Anthony P. Furnary, MD, 9155 SW Barnes Rd, No. 240, Portland, OR 97225 (E-mail: tfurnary@ starrwood.com)

J Thorac Cardiovasc Surg 2003;125: 1007-21

Copyright (C) 2003 by The American Association for Thoracic Surgery

0022-5223/2003\$30.00+0

doi:10.1067/mtc.2003.181
Objective: Diabetes mellitus is a risk factor for death after coronary artery bypass grafting. Its relative risk may be related to the level of perioperative hyperglycemia. We hypothesized that strict glucose control with a continuous insulin infusion in the perioperative period would reduce hospital mortality.

Methods: All patients with diabetes undergoing coronary artery bypass grafting $(\mathrm{n}=3554)$ were treated aggressively with either subcutaneous insulin (1987-1991) or with continuous insulin infusion (1992-2001) for hyperglycemia. Predicted and observed hospital mortalities were compared with both internal and external (Society of Thoracic Surgeons 1996) multivariable risk models.

Results: Observed mortality with continuous insulin infusion $(2.5 \%, \mathrm{n}=$ $65 / 2612)$ was significantly lower than with subcutaneous insulin $(5.3 \%$, $\mathrm{n}=50 / 942, P<.0001)$. Likewise, glucose control was significantly better with continuous insulin infusion $(177 \pm 30 \mathrm{mg} / \mathrm{dL}$ vs $213 \pm 41 \mathrm{mg} / \mathrm{dL}, P<.0001)$. For internal comparison, multivariable analysis showed that continuous insulin infusion was independently protective against death (odds ratio $0.43, P=.001$ ). Conversely, cardiogenic shock, renal failure, reoperation, nonelective operative status, older age, concomitant peripheral or cerebral vascular disease, decreasing ejection fraction, unstable angina, and history of atrial fibrillation increased the risk of death. For external comparison, observed mortality with continuous insulin infusion was significantly less than that predicted by the model (observed/expected ratio $0.63, P<.001$ ). Multivariable analysis revealed that continuous insulin infusion added an independently protective effect against death (odds ratio $0.50, P=.005$ ) to the constellation of risk factors in the Society of Thoracic Surgeons risk model.

Conclusion: Continuous insulin infusion eliminates the incremental increase in inhospital mortality after coronary artery bypass grafting associated with diabetes. The protective effect of continuous insulin infusion may stem from the effective metabolic use of excess glucose to favorably alter pathways of myocardial adenosine triphosphate production. Continuous insulin infusion should become the standard of care for glycometabolic control in patients with diabetes undergoing coronary artery bypass grafting.

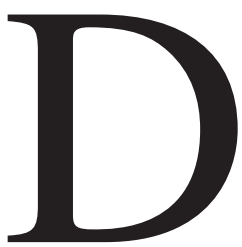
iabetes mellitus (DM) is a well-established risk factor for death after coronary artery bypass grafting $(\mathrm{CABG}) \cdot^{1-3} \mathrm{DM}$ has been recognized as an independent risk factor for CABG-related death since the inception of the Society of Thoracic Surgeons (STS) national risk model in $1991 .{ }^{1} \mathrm{DM}$ is present in $2.5 \%$ of the population in the United States, ${ }^{4}$ but its national prevalence among patients undergoing CABG is as high as $28 \%,{ }^{1}$ making it an important component of the cardiac surgical milieu.

DM is associated with higher incidences of preoperative comorbidities, including obesity, small vessel coronary artery disease, more severe and extensive atherosclerosis, peripheral vascular disease, renal insufficiency, hypertension, and increased rates of life-threatening postoperative infection. Conventional wisdom has held that 
the increase in CABG mortality associated with DM is related to the increased incidence of these comorbid factors associated with DM. ${ }^{3,5,6}$ However, this may not be the case.

In the Diabetes and Insulin-Glucose Infusion in Acute Myocardial Infarction study, Malmberg and associates ${ }^{7}$ showed that survival rates of patients with DM who had acute myocardial infarctions (MIs) were improved when they were treated with insulin infusions designed to achieve normoglycemia. In this study absolute survival rates were improved by $11 \%$ at 1 year and by $15 \%$ at 3.5 years. These authors asserted that glycometabolic control at the time of acute infarction played a leading role in the observed improvement in outcomes. ${ }^{8}$ These findings were corroborated in a prospective randomized trial by the Estudios Cardiologicos Latinoamerica collaborative group, which showed a $66 \%$ relative reduction in post-MI mortality with insulin and glucose metabolic modulation in addition to vessel reperfusion. ${ }^{9}$

Studies with glucose-insulin-potassium (GIK) metabolic modulation in patients undergoing CABG have to date failed to reveal a survival advantage, ${ }^{10,11}$ even for patients with DM. ${ }^{12}$ However, it has been shown that oxidative glycometabolic adenosine triphosphate (ATP) generation is impaired in the ischemic diabetic myocardium. ${ }^{13}$ Patients with DM are known to have increased risk of low cardiac output syndrome and intra-aortic balloon pump use after CABG. We have previously shown that the relative risk of death for a given patient with DM is independently related to the level of perioperative hyperglycemia. ${ }^{14}$ Thus poor glycometabolic control may be detrimental to myocardial function and clinical outcome.

Since January 1987 all patients with DM undergoing heart surgery at Providence St Vincent Medical Center have been enrolled into our ongoing prospective interventional study of the effects of hyperglycemia, and its pharmacologic reduction, on morbidity and mortality. This phase of the project was designed to test the hypothesis that a continuous intravenous insulin infusion (CII) in the perioperative period would reduce mortality among patients with DM undergoing CABG.

\section{Methods}

\section{Patients}

Between January 1987 and December 2001, a total of 14,051 patients underwent isolated CABG at St Vincent Medical Center. All patients with DM who underwent CABG alone, without a concomitant procedure $(n=3554,26 \%$ of all patients undergoing CABG) were included in this study. Patients who underwent CABG combined with other operations (valve replacement or repair, aortic operations, closure of septal defects, or transmyocardial laser revascularization) were excluded from the current study. All isolated on-pump CABG procedures at this institution are performed with short periods of intermittent fibrillation without the use of cardioplegia as a method of myocardial protection. The conditions and conduct of cardiopulmonary bypass remained constant throughout the study period.

All patients in the St Vincent Diabetic Project undergo prospective measurement of blood glucose levels (by finger stick or arterial line drop sample) every 30 minutes to 2 hours in the perioperative period. Average daily glucose levels, along with known preoperative risk factors for morbidity or mortality, were routinely entered into a database for later analysis. These variables included the following:

1. Demographic variables were age, sex, height, weight, and type of preoperative diabetic control (insulin, oral agents, diet, or none).

2. Historical variables were hypertension, congestive heart failure, renal failure, renal insufficiency, chronic obstructive pulmonary disease, pulmonary hypertension, smoking history, current smoking status, recent cerebrovascular accident (within 2 weeks), remote cerebrovascular accident $(>2$ weeks before surgery), peripheral vascular disease, and New York Heart Association functional class.

3. Cardiovascular variables were left main trunk disease, number of diseased vessels, unstable angina, ejection fraction, acute MI, previous MI, timing of previous MI, history of atrial fibrillation, cardiogenic shock, percutaneous transluminal angioplasty, and intra-aortic balloon pump insertion.

4. Preoperative laboratory variables were serum glucose, albumin, hemoglobin $A_{1 C}$, and creatinine levels.

5. Preoperative medications noted were diuretics, digoxin, intravenous nitrates, and steroids.

6. Intraoperative variables were STS operative status (elective, urgent, emergency, or salvage), reoperative cardiac procedures, and cardiopulmonary bypass time.

7. Postoperative variables were total units of blood transfused, prolonged ( $>48$ hours) intubation, inotropic use for longer than 48 hours, epinephrine use, new-onset atrial fibrillation, mediastinitis, and seminal cause of death ${ }^{15}$ (hemorrhage, arrhythmia, pump failure, respiratory failure, neurologic, infection, or renal failure).

\section{Definitions}

Definitions from the STS database committee were used for all variables common with that database. Definitions of other variables unique to this study included the following:

1. DM included all patients admitted to the hospital with a comorbid diagnosis of DM. Patients without a previous diagnosis of DM but with persistently elevated postoperative glucose levels $(>200 \mathrm{mg} / \mathrm{dL})$ and a discharge requirement for pharmacologic glycemic control were also included. These patients were identified as patients with newly diagnosed DM during their admission for CABG.

2. Average postoperative glucose level was the composite average of the daily mean glucose levels from the day of surgery and the first and second postoperative days (PODs). This variable was used as the primary indicator of the pharmacologic effectiveness of hyperglycemic treatment in this study.

3. Mortality or death referred to any in-hospital death occurring at any time during admission for CABG surgery after the start of that surgery. 
4. Cardiac-related mortality referred to all deaths in which arrhythmia or pump failure was identified as the seminal cause of death.

\section{Study Groups}

All patients with DM were divided into two sequential groups according to the type of perioperative glycemic control that they received.

Subcutaneous insulin group. Patients operated on between January 1987 and September 1991, the subcutaneous insulin (SQI) group $(\mathrm{n}=942)$, received subcutaneous insulin injections every 4 hours in a directed attempt to maintain blood glucose levels below $200 \mathrm{mg} / \mathrm{dL}$. Sliding scale dosage of insulin was titrated to each patient's glycemic response during the previous 4 hours. These sliding scale SQI injections were continued every 4 hours throughout the patients' hospital course, even after the resumption of their preoperative glucose control regimen.

CII group. All patients with DM undergoing CABG operated on between October 1991 and December 2001, the CII group ( $\mathrm{n}=$ 2612), received a CII infusion titrated per protocol in the perioperative period (Portland protocol). ${ }^{16}$ The current Portland protocol (Appendix) was implemented in gradual steps designed to maintain patient safety, prevent hypoglycemia, and ensure nursing comfort and compliance. This protocol prescribes insulin initiation, infusion and titration rates, and glucose testing frequency requirements to safely maintain a patient's blood glucose between desired target levels. Between 1991 and 1998, the target glucose range was 150 to $200 \mathrm{mg} / \mathrm{dL}$; in 1999 it was dropped to 125 to 175 $\mathrm{mg} / \mathrm{dL}$ and in 2001 was again lowered to 100 to $150 \mathrm{mg} / \mathrm{dL}$. From 1991 to 1995 , the Portland protocol was used after the operation only in the intensive care unit (ICU) and was stopped when the patient was transferred to the telemetry unit. In January 1996, the protocol was expanded with initiation in the operating room (before sternotomy and after induction of anesthesia, with continuation during cardiopulmonary bypass) and uniform continuation until 7 AM of the third POD, even for patients who had transferred out of the ICU.

Serum potassium levels were maintained between 4.0 and 5.5 $\mathrm{mmol} / \mathrm{L}$ through the administration of exogenous potassium. In the ICU this was accomplished through the intravenous administration of potassium according to a standardized protocol. Oral potassium supplementation was given to maintain these levels once patients were tolerating enteral nutrition and their CII and glucose levels had stabilized.

\section{Data Analysis}

In-hospital mortality was the primary end point of this study. Patient groups were analyzed on an intent-to-treat basis. According to this method, intraoperative and first POD deaths were included in the end point analysis even though those patients did not complete the 3-day SQI or CII treatment protocols. This was considered to be the most rigorous method to test our hypothesis.

An internal logistic regression model was developed to determine the effect of perioperative hyperglycemic treatment method (SQI vs CII) on operative mortality after adjustment for other known preoperative risk factors. The external risk model was taken from the 1996 STS risk algorithm. ${ }^{2}$ The 1996 model was chosen because that year contained the median patient of the study's data set. By means of this nationally recognized risk assessment, all patients were assigned an expected probability of death. Predicted and observed hospital mortalities were then compared, along with the observed/expected risk ratios. The composite STS risk score was calculated as the logit of the probability of death.

Univariate analyses between groups were done with $t$ tests and $\chi^{2}$ analyses. The Bonferroni correction was applied to adjust for multiple comparisons between groups. Stepwise logistic regression was used to produce risk models for hospital death. to measure model discrimination, c-statistics (area under the receiver operating characteristic curve) were used, and the Hosmer-Lemeshow statistic was used to measure calibration. ${ }^{17}$ The purpose was to make internal comparisons rather than to produce a prediction equation for use outside of this data set. Thus all patients were used, rather than separating the data into training and testing subsets or applying shrinkage methods to the coefficients. All statistical analyses were performed with SPSS software (version 10.0; SPSS, Inc, Chicago, Ill).

\section{Results}

Between January 1987 and December 2001, a total of 14,051 patients underwent isolated $\mathrm{CABG}$ at $\mathrm{St}$ Vincent Hospital with an overall mortality of $2.8 \%(n=388 / 14051)$. Of these patients, 3554 (26\%) had DM and were enrolled in this study. The two study groups into which these patients with DM undergoing CABG were divided were slightly heterogeneous (Table 1).

CII was extremely effective in controlling postoperative hyperglycemia. Mean postoperative glucose levels were significantly lower in the CII group than in the SQI group on the day of surgery through the second POD (Table 1). As the Portland CII protocol was implemented in a series of increasingly aggressive steps (see "Methods"), postoperative glycemic control gradually improved (Figure 1).

The postoperative mortality for all patients with DM undergoing CABG in this study was $3.2 \%(n=115 / 3554)$. In-hospital death occurred at a median of 11.2 \pm 13 PODs (range 0-68 days). The overall mortality in the CII group of $2.5 \%(n=65 / 2612)$ was significantly lower than that in the SQI group $(5.3 \%, \mathrm{n}=50 / 942, P<.0001)$. This analysis was performed on an intent-to-treat basis. One could make the argument that those patients who died in the operating room or within 24 hours of surgery $(n=24)$ had "operative catastrophes" and did not survive long enough to derive a benefit from initiation of CII therapy. If our analysis were to be evaluated on an actual treatment (as opposed to intentto-treat) basis, SQI mortality would have been $4.5 \%$ ( $\mathrm{n}=$ 42/934), and CII mortality would have been $1.9 \%$ ( $\mathrm{n}=$ 49/2596, $P<.0001)$.

\section{Cause of Death}

The seminal causes of death for each of the 115 patients who died were pump failure in 54\% $(n=62)$, arrhythmia in $17 \%(\mathrm{n}=20)$, neurologic causes in $19 \%(\mathrm{n}=22)$, respiratory failure in $5 \%(n=6)$, renal failure in $3 \%(n=3)$, 
TABLE 1. Characteristics of study groups

\begin{tabular}{|c|c|c|c|c|}
\hline Variable & All & SOI & CII & $P$ value \\
\hline No. of patients & 3554 & 942 & 2612 & \\
\hline \multicolumn{5}{|l|}{ Preoperative } \\
\hline Age $(y$, mean $\pm S D)$ & $64 \pm 10$ & $65 \pm 9$ & $64 \pm 10$ & \\
\hline Male (No.) & $2316(65 \%)$ & $603(64.0 \%)$ & $1713(65 \%)$ & \\
\hline \multicolumn{5}{|l|}{ Diabetic control (No.) } \\
\hline Insulin & $1171(33 \%)$ & $314(33 \%)$ & $857(33 \%)$ & \\
\hline Oral agent & $1784(50 \%)$ & $455(48 \%)$ & $1329(51 \%)$ & \\
\hline Diet only & $405(11 \%)$ & $108(12 \%)$ & $297(11 \%)$ & \\
\hline No prescription & $194(6 \%)$ & $65(7 \%)$ & $129(5 \%)$ & \\
\hline Smoking (No.) & $880(25 \%)$ & $150(16 \%)$ & $730(28 \%)$ & $<.001$ \\
\hline Hypertension (No.) & $2426(68 \%)$ & $545(58 \%)$ & $1881(72 \%)$ & $<.001$ \\
\hline Congestive heart failure (No.) & $738(21 \%)$ & $222(24 \%)$ & $516(20 \%)$ & \\
\hline Renal failure (No.) & $141(4.0 \%)$ & $47(5.1 \%)$ & $94(3.6 \%)$ & \\
\hline Renal insufficiency (No.) & $234(6.6 \%)$ & $29(3.1 \%)$ & $205(7.9 \%)$ & $<.001$ \\
\hline Peripheral vascular disease (No.) & $649(18 \%)$ & $160(17 \%)$ & $489(19 \%)$ & \\
\hline Cerebral vascular disease (No.) & $315(9 \%)$ & $97(11 \%)$ & $218(9 \%)$ & \\
\hline Chronic obstructive pulmonary disease (No.) & $365(11 \%)$ & $74(8 \%)$ & $291(11 \%)$ & \\
\hline Pulmonary hypertension (No.) & $71(2.0 \%)$ & $14(1.5 \%)$ & $58(2.2 \%)$ & \\
\hline Body mass index $\left(\mathrm{kg} / \mathrm{m}^{2}\right.$, mean $\left.\pm \mathrm{SD}\right)$ & $29.7 \pm 5.6$ & $28.4 \pm 5.1$ & $30.2 \pm 5.7$ & $<.001$ \\
\hline Ejection fraction $(\%$, mean \pm SD) & $54.9 \pm 16$ & $53.2 \pm 17$ & $55.4 \pm 15$ & \\
\hline Preoperative intra-aortic balloon pump (No.) & $63(1.8 \%)$ & $23(2.6 \%)$ & $40(1.6 \%)$ & \\
\hline No. of diseased vessels (mean \pm SD) & $2.6 \pm 0.6$ & $2.6 \pm 0.6$ & $2.6 \pm 0.6$ & \\
\hline Left main trunk $>50 \%$ (No.) & $545(16 \%)$ & $117(13 \%)$ & $428(17 \%)$ & \\
\hline Previous MI (No.) & $1926(55 \%)$ & $541(58 \%)$ & $1385(53 \%)$ & \\
\hline Acute MI (No.) & $747(22 \%)$ & $201(22 \%)$ & $546(21 \%)$ & \\
\hline Percutaneous transluminal angioplasty (No.) & $71(2.1 \%)$ & $27(3.0 \%)$ & $44(1.7 \%)$ & \\
\hline Intravenous nitrates (No.) & $745(22 \%)$ & $165(19 \%)$ & $580(23 \%)$ & \\
\hline Digoxin (No.) & $504(15 \%)$ & $185(21 \%)$ & $319(12 \%)$ & $<.001$ \\
\hline Diuretics (No.) & $1163(34 \%)$ & $363(41 \%)$ & $800(31 \%)$ & $<.001$ \\
\hline Steroids (No.) & $154(4.3 \%)$ & $33(3.5 \%)$ & $121(4.6 \%)$ & \\
\hline Albumin $(\mathrm{g} / \mathrm{dL}$, mean $\pm \mathrm{SD})$ & $3.7 \pm 0.5$ & $3.9 \pm 0.6$ & $3.6 \pm 0.5$ & $<.001$ \\
\hline Admission glucose $(\mathrm{mg} / \mathrm{dL}$, mean $\pm \mathrm{SD})$ & $171 \pm 60$ & $176 \pm 63$ & $170 \pm 59$ & \\
\hline \multicolumn{5}{|l|}{ Perioperative } \\
\hline \multicolumn{5}{|l|}{ Postoperative blood glucose $(\mathrm{mg} / \mathrm{dL}$, mean $\pm \mathrm{SD})$} \\
\hline Average & $187 \pm 37$ & $214 \pm 41$ & $177 \pm 30$ & $<.001$ \\
\hline Day of surgery & $201 \pm 53$ & $242 \pm 61$ & $187 \pm 41$ & $<.001$ \\
\hline POD 1 & $181 \pm 34$ & $205 \pm 36$ & $173 \pm 28$ & $<.001$ \\
\hline POD 2 & $181 \pm 40$ & $195 \pm 39$ & $176 \pm 39$ & $<.001$ \\
\hline Mediastinitis (No.) & $33(0.9 \%)$ & $17(1.8 \%)$ & $16(0.6 \%)$ & .05 \\
\hline Reoperative sternotomy (No.) & $416(12 \%$ & $138(15 \%$ & $278(11 \%$ & .04 \\
\hline Urgency of operation (No.) & & & & .002 \\
\hline Elective & $1866(53 \%)$ & $469(50 \%)$ & $1397(53 \%)$ & \\
\hline Urgent & $146(41 \%)$ & $400(42 \%)$ & $1067(41 \%)$ & \\
\hline Emergency & $187(5 \%)$ & $52(5.5 \%)$ & $135(5.2 \%)$ & \\
\hline Salvage & $38(1 \%)$ & $23(2.4 \%)$ & $15(0.6 \%)$ & \\
\hline Internal thoracic artery use (No.) & & & & $<.01$ \\
\hline 0 & $929(26 \%)$ & $352(37 \%)$ & $577(22 \%)$ & \\
\hline 1 & $2571(72 \%)$ & $589(63 \%)$ & $1982(76 \%)$ & \\
\hline 2 & $52(1.5 \%)$ & $1(0.1 \%)$ & $51(2.0 \%)$ & \\
\hline Off-pump procedure & $37(1.0 \%)$ & $0(0 \%)$ & $37(1.4 \%)$ & .01 \\
\hline Cardiopulmonary bypass time $(\min$, mean $\pm \mathrm{SD})$ & $80.5 \pm 34$ & $85.2 \pm 39$ & $78.7 \pm 32$ & $<.001$ \\
\hline Transfused packed red blood cells (units, mean \pm SD) & $1.8 \pm 5.9$ & $2.2 \pm 2.9$ & $1.6 \pm 6.7$ & \\
\hline Epinephrine use (No.) & $137(3.9 \%)$ & $33(3.5 \%)$ & $104(4.0 \%)$ & \\
\hline Inotropes $>48 \mathrm{~h}$ (No.) & $294(8.3 \%)$ & $99(10.5 \%)$ & $195(7.5 \%)$ & \\
\hline Low cardiac output syndrome & $370(11 \%)$ & $115(13 \%)$ & $225(10 \%)$ & .09 \\
\hline Ventilation $>48 \mathrm{~h}$ (No.) & $192(5.4 \%)$ & $61(6.5 \%)$ & $131(5.0 \%)$ & \\
\hline New-onset atrial fibrillation & $729(21 \%)$ & $253(27 \%)$ & $476(18 \%)$ & $<.001$ \\
\hline Length of stay ( $d$, mean $\pm S D)$ & $8.6 \pm 5.7$ & $10.4 \pm 6.6$ & $8.0 \pm 5.3$ & $<.001$ \\
\hline Observed deaths (No.) & $115(3.2 \%)$ & $53(5.3 \%)$ & $67(2.5 \%)$ & .001 \\
\hline STS-predicted deaths (No.) & 4 & 4.7 & 3.8 & \\
\hline
\end{tabular}




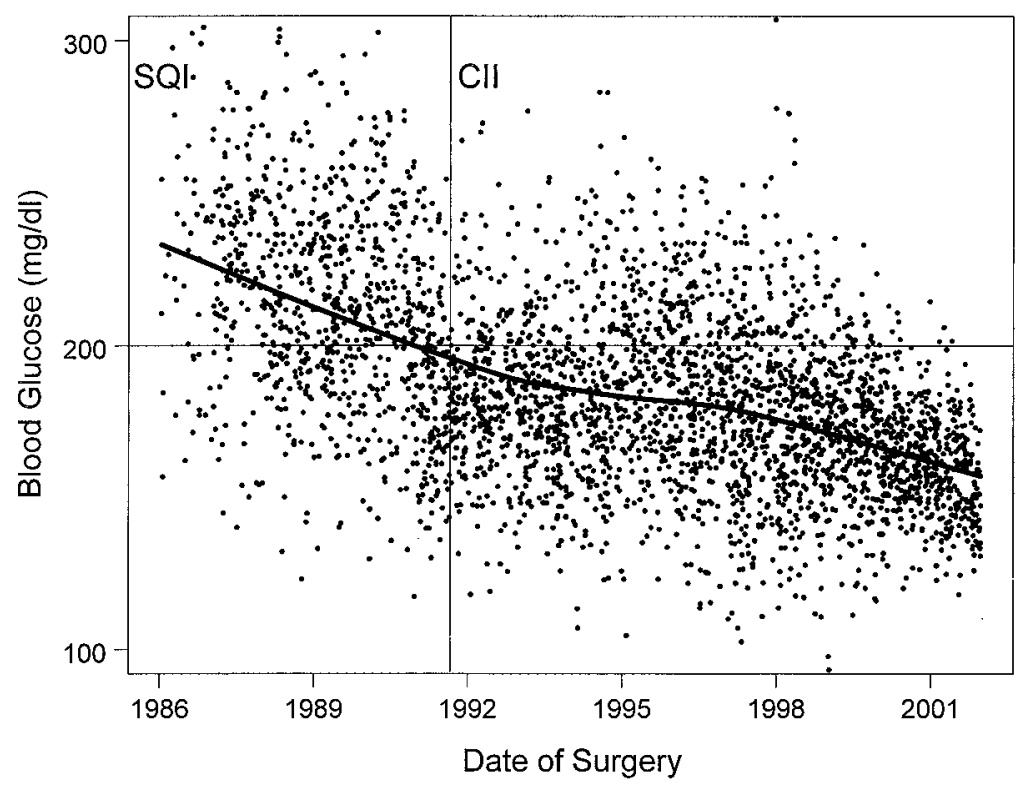

Figure 1. Scattergram of average postoperative glucose levels of all 3554 patients with DM undergoing CABG according to date of surgery. Smoothed local regression (Loess) curve is superimposed. Initiation of Portland protocol is marked by vertical line. Note gradual reduction of glucose levels with time.

hemorrhage in $1 \%(\mathrm{n}=1)$, and infection in $1 \%(\mathrm{n}=1)$. Cardiac-related causes accounted for most $(71 \%, \mathrm{n}=85 /$ 120) of the deaths in this series. Cardiac-related mortality was significantly greater in the SQI group $(4.2 \%, \mathrm{n}=$ 40/942) than in the CII group $(1.6 \%, \mathrm{n}=42 / 2612 ; P<$ $.001)$, implicating a myocardial mechanism of action for CII. There was no difference between the two groups in the incidence of deaths from noncardiac causes $(1.1 \%$ [n $=$ $10 / 942]$ vs $0.9 \%$ [n $=23 / 2612], P=.5)$. Conversely stated, the reduction in operative mortality seen with CII was accounted for solely by a reduction in cardiac-related deaths.

An analysis of mortality according to glucose quantile is presented in Figure 2. This shows a highly significant relationship $(P<.001)$ between mortality and postoperative glucose levels rising above $175 \mathrm{mg} / \mathrm{dL}$. Figure 2 also reveals that the increase in overall mortality was principally accounted for by an increase in cardiac-related mortality. Non-cardiac-related mortality did not increase as postoperative glucose levels rose $(P=.9)$.

\section{Internal Multivariable Analysis of Mortality}

An internal logistic regression model that was based solely on these data was developed to determine the effect of CII on operative mortality after adjustment for other known preoperative risk factors (Table 2). Treatment of hyperglycemia with CII independently reduced the odds of mortality by a factor of $57 \%$ (odds ratio $0.43, P=.001$ ). Cardiogenic shock, renal failure, reoperation, increasing urgency of op- eration, increasing age, a history of peripheral or cerebral vascular disease, decreasing ejection fraction, unstable angina, and a history of atrial fibrillation were all found to increase the risk of death. The c-statistic for this model was 0.874 , indicating high predictability for post-CABG mortality among patients with DM.

Several other preoperative variables, known from other logistic regression models to predispose toward death after CABG, did not enter this model. These included gender $(P=.8)$, congestive heart failure $(P=.2)$, left main trunk disease $(P=.2)$, previous MI $(P=.8)$, acute MI $(P=.5)$, preoperative intra-aortic balloon pump $(P=.5)$, previous percutaneous transluminal angioplasty $(P=.3)$, chronic obstructive pulmonary disease $(P=.2)$, intravenous nitrates $(P=.4)$, diuretic $(P=.4)$, digoxin $(P=.2)$, pulmonary hypertension $(P=.3)$, and steroid use $(P=.9)$. Importantly, neither date of operation $(P=.6)$ nor year of operation $(P=.8)$ was found to independently influence mortality.

Postoperative epinephrine use and deep sternal infections have both been shown to increase glucose levels and mortality. They are, however, not "preordained" variables and should not be used to infer risk in predictive models. Nonetheless, when added into the internal model they were both highly significant $(P<.001)$. When these additions were made to this model, they actually increased the protective significance of CII $(P<.001$, odds ratio 0.36$)$, with history of atrial fibrillation becoming nonsignificant and ejection 


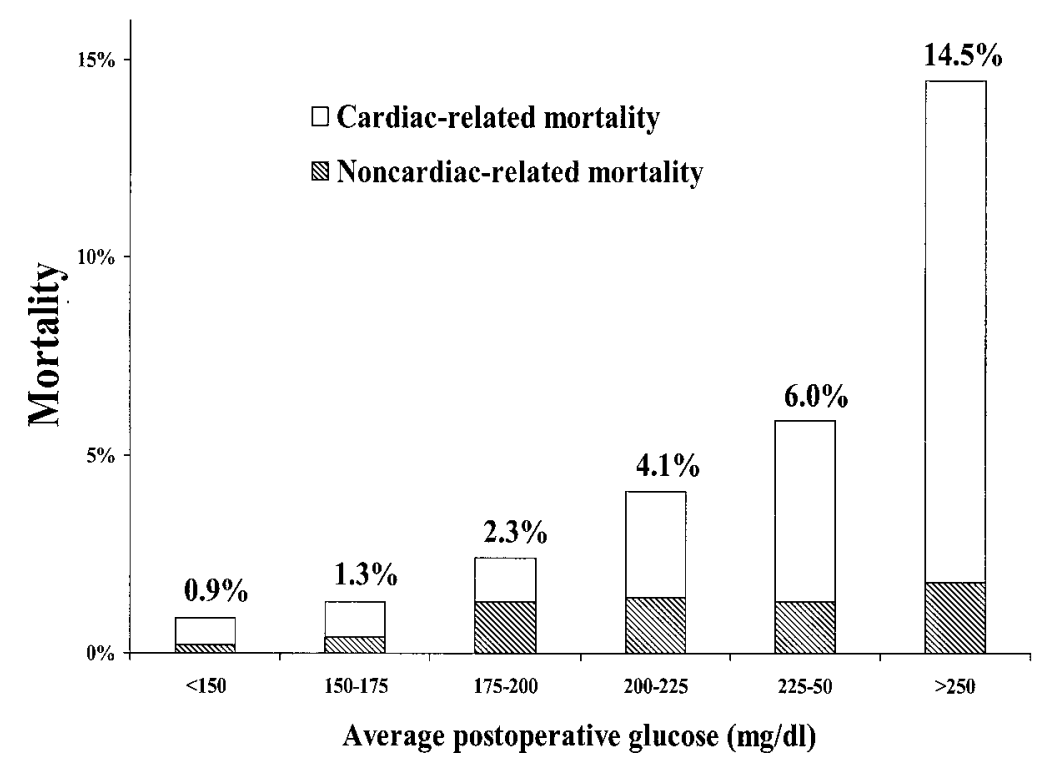

Figure 2. Mortality among patients with DM undergoing CABG by glucose quantile. Total bar heights represent overall mortality in each quantile. Note increase in overall mortality is overwhelmingly accounted for by increase in cardiac-related mortality.

TABLE 2. Multivariable analysis of mortality in patients with DM undergoing CABG $(\mathbf{N}=2933)$

\begin{tabular}{lccc}
\hline & & \multicolumn{2}{c}{ Odds ratio } \\
\cline { 2 - 4 } Variable & $\boldsymbol{P}$ value & Value & 95\% confidence interval \\
\hline Insulin infusion & .001 & 0.43 & $0.26-0.70$ \\
Cardiogenic shock & $<.001$ & 5.5 & $2.4-12.8$ \\
Renal failure & $<.001$ & 3.5 & $1.8-6.9$ \\
Reoperation & $<.001$ & 2.8 & $1.7-5.0$ \\
Operative status & $<.001$ & $1.7-3.3$ \\
Age (per 1-y increment) & .001 & $1.02-1.08$ \\
Ejection fraction (per 1\% increment) & .001 & 1.05 & $0.96-0.99$ \\
Unstable angina & .001 & 0.98 & $1.6-6.5$ \\
History of peripheral or cerebral vascular disease & .002 & 3.2 & $1.3-3.5$ \\
History of atrial fibrillation & .05 & 2.1 & $1.01-3.9$ \\
\hline
\end{tabular}

Area under receiver operating characteristic curve, 0.874 ; Hosmer-Lemeshow goodness-of-fit, $P=.6$.

fraction, cardiogenic shock, and reoperation all slightly decreasing in significance. The c-statistic of this postoperatively enhanced model was improved to 0.9 .

When the continuous variable of average postoperative blood glucose level was entered into the logistic regression as a potentially more accurate reflection of glycometabolic control, it displaced the categoric variable of CII from the equation, leaving all other variables in place $(P<.001$, odds ratio 1.018 per $1 \mathrm{mg} / \mathrm{dL}$, c-statistic 0.886 ). This again implies that CII is exerting a protective effect on mortality through a direct reduction of hyperglycemia, which may reflect an underlying detrimental metabolic defect within the postischemic diabetic myocardium.

The individual daily average glucose levels from the day of surgery $(P<.003$, odds ratio 1.006 per $1 \mathrm{mg} / \mathrm{dL})$ and from the first $(P<.001$, odds ratio 1.013 per $1 \mathrm{mg} / \mathrm{dL})$ and second $(P<.015$, odds ratio $=1.018$ per $1 \mathrm{mg} / \mathrm{dL})$ PODs were each significant independent predictors of death when entered into the model in lieu of the composite 3-day average glucose. Glucose levels on the third POD did not have a significant independent effect on mortality $(P=.1)$. This further implies that the protective effects of CII are in play at least until the third POD.

The excluded subset of 340 patients who underwent CABG combined with valve repair or replacement had blood cardioplegia delivery as a method of myocardial protection. Mortality with CII was $7.4 \%$, compared with $12.7 \%$ with SQI. Logistic regression analysis in this population revealed a similar protective effect of CII against death (odds ratio 0.48$)$, although it was not significant $(P=$ 
TABLE 3. STS predicted operative risk ${ }^{2}$ versus observed mortality ( $N=2834)$

\begin{tabular}{lccc}
\hline & Sol & CII & $\begin{array}{c}\boldsymbol{P} \\
\text { value* }\end{array}$ \\
\hline Observed deaths & & & $<.001$ \\
$\quad$ No. & $36 / 673$ & $51 / 2161$ & \\
$\quad \%$ & 5.35 & 2.36 & \\
$\begin{array}{l}\text { Predicted deaths (\%) } \\
\text { Observed:expected ratio }\end{array}$ & $4.68 \pm 7.8$ & $3.77 \pm 5.5$ & .2 \\
$\quad$ Value & 1.14 & & $<.001$ \\
$\quad \begin{array}{l}\text { 95\% Confidence } \\
\text { interval }\end{array}$ & $0.87-1.51$ & $0.45-0.87$ & \\
$P$ valuet & & & \\
\hline
\end{tabular}

*SOl versus CII.

tObserved versus expected.

.11) because of the small sample size. This suggests that the effects of CII are not idiosyncratically related to our method of myocardial protection for patients undergoing isolated CABG.

\section{External Multivariable Analysis of Mortality}

To determine the effect of CII on risk-adjusted mortality, the predicted operative risk derived from the 1996 STS risk algorithm ${ }^{2}$ was calculated for every patient with all such variables available $(\mathrm{n}=2834)$ and compared with observed mortality (Table 3). STS-predicted mortality for the CII group was lower than that for the SQI group. Observed mortality with SQI did not significantly differ from that predicted by the STS model. However, observed mortality with CII was significantly less than predicted. These data show a $36 \%$ reduction in the expected mortality resulting from CII.

To further confirm the significance of the reductive effect of CII on risk-adjusted mortality, the STS composite risk score, was entered into a new multivariable analysis of death along with CII. Both variables were significant (STS risk score odds ratio $3.3,95 \%$ confidence interval 2.7-4.0, $P<.001$, CII odds ratio $0.50,95 \%$ confidence interval $0.30-0.76, P=.005$, c-statistic 0.839). Thus CII added a protective effect against death to the constellation of risk factors already in the STS risk model. This external model suggests that CII conferred a 50\% reduction in the riskadjusted mortality of patients with DM undergoing CABG. This would translate into 21 lives saved for every 1000 patients for whom CII is effectively implemented.

The surrogate variable of average postoperative glucose level was again found to displace the categoric variable of CII from the external model $(P<.001$, odds ratio 1.02 per $1 \mathrm{mg} / \mathrm{dL}, \mathrm{c}$-statistic 0.853 ), again implicating a glycometabolic mechanistic effect for CII. To account for the confounding influences of time and sequential controls, the continuous variable of surgery date was once again forced into the equation and found not to be significant $(P=.9)$.

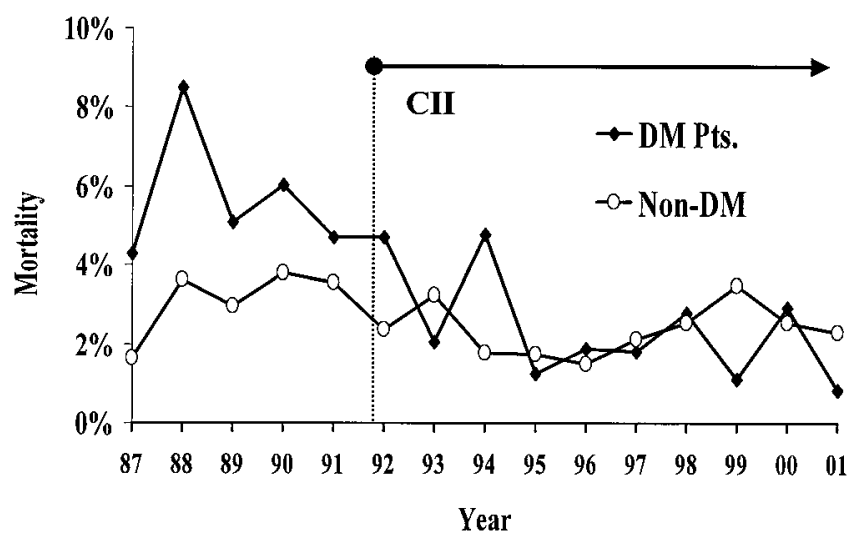

Figure 3. Annualized mortality in all patients undergoing CABG (with DM, filled diamonds, vs without DM, open circles) during study years. Mortality in group without DM has not changed with time. However, mortality in group with DM has decreased dramatically. There is currently (1995-2001) no statistical difference between these two groups.

The temporal effect of the Portland CII protocol on mortality among patients with DM undergoing CABG is illustrated in Figure 3, which depicts the annualized operative mortality for all patients undergoing $\mathrm{CABG}$ at our institution. Mortality among patients with DM undergoing CABG has fallen significantly since CII implementation in 1992. Perioperative mortality among patients without DM undergoing $\mathrm{CABG}$ has not changed during the same period (slope of $0.9, P=.4$ ). There are now no significant differences in operative mortality between patients with and without DM undergoing $\mathrm{CABG}$ at this institution.

\section{Discussion}

The principal finding of this study was that CII for patients with DM undergoing CABG independently reduced perioperative absolute mortality by $57 \%$ and risk-adjusted mortality by $50 \%$. Improved survival with CII came about exclusively through a reduction in cardiac-related deaths. CII had the resultant effect of eliminating the incremental effect of DM on mortality risk in our population of patients undergoing CABG (Figure 3).

The limitations of this study should be noted. First, this was a nonrandomized study that compared sequential groups of patients. Second, the use of asynchronous controls resulted in heterogeneous study groups. This makes direct comparison of the primary end point "death" difficult because the accompanying concomitant risks were not equally dispersed. Finally, the prolonged time frame of this study induces further questions concerning temporal technical biases that are difficult to measure.

Because of the automated and aggressive nature of the Portland CII protocol, it was not feasible for us to conduct this study in a synchronously randomized fashion. Nursing 
comfort and confidence with the perceived safety of CII titration in patients with what was considered to be euglycemia or mild hyperglycemia was programmatically difficult. Nursing and administrative concerns about iatrogenically induced hypoglycemia in this high-visibility patient population had first to be assuaged. This was accomplished gradually, first in the ICU and then on the telemetry floor, through rigorous and repeated in-service training conferences. When the protocol was finally functioning smoothly in the desired units, target glucose levels were then gradually lowered. As can be seen in Figure 1, tight perioperative glycemic control took years for full implementation and achievement. Once the other beneficial effects of tight glucose control on patient outcomes (decreased wound infections, decreased length of stay) became known, ${ }^{14,16,18}$ both we and the institutional review board considered a synchronously randomized study with SQI control subjects unethical. Thus the nonrandomized nature of this study at this institution is and must remain a statistical design flaw to which some reviewers may object.

Although these technical limitations cannot be fully abrogated, we sought to minimize temporal bias and heterogeneity through appropriately sound statistical methods. Multivariable analyses serve well to smooth out baseline constitutional differences between groups. ${ }^{19,20}$ A well-accepted, nationally derived, external risk model $^{2}$ was used to normalize both constitutional makeup and temporal biases. All multivariable analyses, both internal and external, continued to reveal the protective significance of CII.

The long time frame of this study was necessary to accumulate enough outcome data from patients with DM undergoing $\mathrm{CABG}$ to power the study effectively. We accumulated an average of 237 patients with DM undergoing CABG per year toward the goal of 4000 patients, the number that would have been required to detect a $30 \%$ decrease in an overall mortality of $5 \%$.

Temporal bias is further excluded by the following facts: (1) date of surgery had no significant effect on either multivariable model and (2) mortality among patients without DM undergoing CABG did not change with time. Nonetheless, we cannot rule out a conglomeration of minute improvements in operative technique during the 15-year study period that may have contributed to a decline in DMassociated mortality.

Analysis of average postoperative glucose level does not carry with it the biases of nonrandomization and asynchronous controls. Rather, it is a direct measure of the underlying glycometabolic state of the myocardium that in itself is devoid of group selection and temporal bias. Thus it is important to note that in both multivariable models mortality was also independently linked with average postoperative glucose level. This relationship held regardless of the study group into which the patient was entered.
This study was not intended to definitively establish a biochemical mechanism of action for the mortality-reducing effects of CII. However, on the basis of previously published literature, we have theorized that alterations in myocardial metabolism in ischemic patients with DM undergoing $\mathrm{CABG}$ are detrimental, whereas insulin-enhanced alterations in myocardial energy formation are one of the potential mechanisms for the favorable effects of $\mathrm{CII}$ on mortality. The following subsections of the discussion are offered as an exposition of previously published works in this field that support this theory.

\section{Normal Myocardial Energetics}

The myocardium has been described as an "omnivore" because it is able to use any of several substrates for the production of ATP to power the continuous cycle of ventricular contraction and relaxation. The known substrates include free fatty acids (FFAs), glucose, pyruvate, lactate, ketones, and even amino acids. ${ }^{21}$ In normal nondiabetic, nonischemic myocardium, $60 \%$ of ATP production is derived from lipolysis and $\beta$-oxidation of palmitate or FFAs, whereas $35 \%$ is derived from glycolytic sources. ${ }^{22}$ Both glycolysis and FFA $\beta$-oxidation eventually produce acetyl coenzyme A. This is the primary substrate that produces hydrogen ions for oxidative phosphorylation through the Krebs cycle in the mitochondria. Feedback mechanisms related to the concentration of acetyl coenzyme $\mathrm{A}$ in the mitochondria ensure a balance between these two pathways. . $^{23,24}$

Anerobic glycolysis occurs in the cytosol and produces pyruvate while regenerating cytosolic ATP, which is critical for the maintenance of cell membrane integrity. ${ }^{25}$ It is also used to phosphorylate extracellular glucose for active transport into the cytosol and subsequently on to glycolysis. ${ }^{26}$ Pyruvate passively diffuses into the mitochondria, where it is decarboxylated to acetyl coenzyme A by pyruvate dehydrogenase complex (PDH). ${ }^{24}$ Oxidative glycolysis can then be completed by the Krebs cycle. In the absence of insulin, PDH activity decreases in the mitochondria, pyruvate builds up in the cytosol, and excess pyruvate is converted to lactate. The reduction and decarboxylation of pyruvate by PDH thus becomes the rate-limiting step for further oxidative glycolysis. Lipolysis-derived FFAs are actively transported into the mitochondria, where they undergo $\beta$-oxidation to produce acetyl coenzyme A. Increased levels of FFA-derived acetyl coenzyme A inhibit PDH and thus inhibit oxidative glycolysis. ${ }^{24}$

\section{Alterations in Myocardial Energetics in Patients With DM Undergoing CABG}

In patients with $\mathrm{DM}$ undergoing $\mathrm{CABG}$, myocardial metabolism is negatively altered by both the ischemic and diabetic pathologic states. In patients without DM, the supply of 
molecular oxygen is limited during periods of ischemia. FFA oxidation is inhibited, and oxygen-efficient glycolytic ATP production predominates. In patients with poorly controlled DM undergoing $\mathrm{CABG}$, however, this is not possible because glycolysis is hormonally inhibited and lipolysis is paradoxically enhanced.

Deficiencies in insulin bioavailability increase serum concentrations and myocardial use of FFAs, further inhibiting glucose use. Serum glucose levels consequently rise in proportion to the underlying glycometabolic defect in the cells. A paucity of bioavailable insulin in the cell also slows phosphorylation of glucose and fails to activate $\mathrm{PDH} .{ }^{27}$ Glycolysis is thus inhibited in diabetic myocardium, and FFA oxidation is paradoxically and detrimentally activated. Ischemic cardiomyocytes must now derive as much as $90 \%$ of their energy from FFA metabolism. ${ }^{13}$ Because of limited oxidative capacity, the FFAs taken up by the myocardium are not completely metabolized. FFAs and their partially $\beta$-oxidized intermediates accumulate in the myocardium. These compounds are known to decrease contractility and increase the incidence of ventricular arrhythmias. ${ }^{28,29} \mathrm{Al}-$ though FFAs produce more ATP than does glucose does during complete aerobic oxidation, they do so at the expense of a higher rate of oxygen consumption. ${ }^{25}$ This further increases myocardial oxygen consumption and exacerbates cellular ischemia at a time when oxygen supply is limited.

Glycolysis-derived cytosolic ATP preferentially supports cell membrane ion transport and hence helps to preserve cellular integrity. ${ }^{25}$ Even after successful revascularization and reperfusion of the underlying ischemia, postoperative deficiencies in glucose metabolism persist in patients with poorly controlled DM. The persistent lack of glycolysisderived ATP prolongs membrane destabilization and leads to increased cellular edema and arrhythmogenic potential. ${ }^{28}$

In summary, serum glucose level may act as a "fuel gauge" that varies inversely with the ability of the cardiomyocyte to effectively absorb and use that fuel. In patients with poorly controlled DM undergoing CABG, glycolysis is inhibited, serum glucose is elevated, FFA metabolism is paradoxically activated, and FFA intermediates accumulate in myocardial cells. The serum glucose level thus reveals the underlying glycometabolic state of the myocardium to the astute clinician.

\section{Mechanism of Action of CII}

The administration of insulin to the hyperglycemic patient with DM undergoing CABG reverses the aforementioned metabolic deficiencies. Exogenous intravenous insulin causes both intracellular and extracellular insulin levels to rise. As intracellular insulin rises, PDH is activated. ${ }^{30}$ As mitochondrial pyruvate levels fall, cytosolic pyruvate is depleted by diffusion, opening up the pathway for increased cytosolic glycolysis. Glycolysis, again stimulated by insulin, replenishes cytosolic ATP, which is in turn used to stabilize cellular membranes, phosphorylate extracellular glucose for transport into the cell, and facilitate membrane ion transport. These processes are crucial to endothelial, vascular smooth muscle, and myocardial cellular integrity. ${ }^{31}$ Blood glucose levels are in turn lowered as myocardial glucose uptake is enhanced. ${ }^{27}$ Preservation of myocyte, endothelial and smooth muscle cell membranes results in decreased cellular edema, reduced microvascular compression, and prevention of the no-reflow phenomenon that may occur during reperfusion. ${ }^{32}$ The preservation of endothelial integrity and vascular smooth muscle function may also improve myocardial function by increasing native myocardial perfusion and by lowering systemic and pulmonary afterload resistances. ${ }^{31}$

Intracellular glycerol esterifies intracellular FFAs, preventing them from being transported into the mitochondria. ${ }^{32}$ In addition, the increase in mitochondrial acetyl coenzyme A derived from active glycolysis inhibits the carnitine-assisted transport of FFAs into the mitochondria. ${ }^{24}$ This explains how increases in glucose oxidation are able to downwardly regulate myocardial FFA oxidation. Myocardial oxygen consumption is thus decreased by shutting down the $\beta$-oxidation of FFAs. Accumulation of the negatively inotropic intermediaries of FFA oxidation ceases, ${ }^{28}$ free radical formation stops, and myocardial efficiency and function improve. ${ }^{34}$ Insulin may further protect subcellular function by serving as a scavenger of free radicals generated during the ischemia-reperfusion process. ${ }^{34}$ Insulin thus directly enhances glycolysis, mediates active transport of phosphorylated glucose across the cell membrane, and inhibits further lipolysis, preventing buildup of toxic intermediates. ${ }^{27,35}$

The clinical effects of the CII protocol may also be related to other sequelae of insulin administration on the myocardium, such as increased uptake of potassium or magnesium into the myocyte. It is also possible that some of the beneficial outcomes seen with CII are related to its effects in tissues other than the heart, such as improved energetics of skeletal muscle, lower circulating lactate levels during the interval on cardiopulmonary bypass, and improved endothelial function, as mentioned previously. It is important to note that our study provides no data on serum insulin levels, FFA levels, intracellular metabolite levels, myocardial ATP levels, enzyme activity levels, or glycolic rates. Therefore a definitive, indirect relationship between serum glucose levels and myocardial glycolysis rates is not proved by our study.

\section{Clinical Evidence}

There is, however, abundant clinical evidence that glycometabolic processes indeed play a role in critically ill patients. Not only is DM a risk factor for CABG mortality, ${ }^{1-3}$ it also independently predicts higher incidences of postop- 
erative arrhythmias, low cardiac output syndrome, and intra-aortic balloon pump use. ${ }^{36-38}$

Glucose does appear to be a superior substrate during periods of myocardial ischemia. ${ }^{32}$ However, that substrate must be made biologically available by an adequate supply of insulin. In the Diabetes and Insulin-Glucose Infusion in Acute Myocardial Infarction study, Malmberg and associates $^{7}$ demonstrated that intensive glycometabolic control with CII after MI in patients with DM led to improved long-term survival. ${ }^{7}$ The Estudios Cardiologicos Latinoamerica study group demonstrated a $66 \%$ reduction in acute post-MI mortality with GIK modulation in addition to reperfusion. ${ }^{9}$

GIK solutions in acutely ischemic myocardium have been shown to enhance contractility, ${ }^{39}$ decrease arrhythmias, and decrease myocardial oxygen consumption. ${ }^{27}$ Rao and colleagues ${ }^{11}$ have shown that insulin-enhanced cardioplegia improves post-arrest stroke work and cardiac indices. In addition, it hastens the return of myocardial oxygen extraction to baseline after cardioplegic arrest.

Lazar and associates' extensive clinical and experimental work with $\mathrm{GIK}^{40}$ has shown us that this therapy limits postischemic tissue necrosis, infarct size, and acidosis, and it prevents myocardial stunning. Clinically this has resulted in increased cardiac indices, decreased weight gain, shortened ventilator times, and reduced atrial arrhythmias among patients undergoing CABG who require urgent revascularization for ongoing ischemia. ${ }^{10}$ In patients with DM undergoing CABG, GIK had the additional effect of shortening hospital stay. ${ }^{12}$ Recently GIK solutions have also been shown to improve left ventricular contractility and ventriculoarterial coupling in diabetic sheep. ${ }^{41}$

Although they were not the primary end points of the current study, there were univariate reductions in the incidences of new-onset atrial fibrillation and low cardiac output syndrome with the use of CII (Table 1). These findings further support our theoretic assertion that alterations in glycometabolic function play an etiologic role in the outcome alterations that we have seen.

\section{Portland CII Protocol}

We propose that the glycometabolic state of the cardiomyocyte is the final and true variable that directly affects outcomes in patients with DM undergoing CABG. The serum glucose level merely illustrates the level of the underlying glycometabolic deficiency for the clinician. The Portland CII protocol is a directed therapy designed to normalize the glycometabolic state of the myocardium in patients with DM.

The Portland CII protocol is similar to GIK therapy in that insulin and potassium are iatrogenically administered to safely enhance glucose use. However, the Portland CII protocol is an insulin therapy that is precisely tailored to correct the specific glycometabolic defect that exists in each patient. Every patient with DM has a unique degree of glucose-insulin mismatch and an accompanying unique glycometabolic deficiency. Exogenous glucose is not "force fed" to the cells in an attempt to turbo charge ATP production and reduce FFA use, as it is with GIK regimens in patients without DM. Rather, excess endogenous glucose is used as myocardial substrate. Thus the clinician can directly monitor the cellular metabolic effectiveness of CII therapy by monitoring serum glucose levels. The induced physiologic hyperinsulinemia alleviates the glycolytic deficiency in direct proportion to its severity.

Previous studies have failed to demonstrate a clinical survival benefit from the administration of GIK to patients undergoing CABG. ${ }^{10,12}$ There are several potential methodologic explanations. Most clinical GIK studies have been done in ischemic patients without DM. Unlike patients with $\mathrm{DM}$ undergoing $\mathrm{CABG}$, these patients do not have a persistent and prolonged glycometabolic defect after reperfusion. Even the beneficial effects of insulin-enhanced cardioplegia are dissipated by 8 hours in patients without DM undergoing CABG. ${ }^{11}$ Furthermore, with the addition of exogenous glucose there is no clinical mechanism of monitoring the effectiveness of cellular glucose loading to increase oxidative glycolysis. In addition to applying a nontailored glycometabolic therapy to patients without DM, most studies have been underpowered to detect small differences in mortality.

In Lazar and associates' study on patients with DM, ${ }^{12}$ GIK was used in the operating room and only for 12 hours after the operation in the ICU. Because glycolysis-derived ATP is critical to myocardial, endothelial, and smooth muscle membrane stability, whereas accumulation of FFA intermediates is detrimental to these cells' function, ${ }^{32}$ we believe that glucose metabolism should be maintained at optimum levels for at least the first 2 PODs. This has now been elucidated by our findings that daily average glucose levels are significant independent predictors of death until the third POD, when their significance ceases. Maintenance of tight glycometabolic control throughout this period of maximum postoperative cellular edema should serve to stabilize cell membranes, enhance endothelial function, and reduce further fluid accumulation.

There is one previously published study showing a survival advantage with CII in postoperative surgical patients. ${ }^{42}$ In that study, CII was used in a heterogeneous group of hyperglycemic patients in an ICU setting. Insulin was titrated to a euglycemic target of 80 to $110 \mathrm{mg} / \mathrm{dL}$, and exogenous glucose was not used. Most interestingly, the survival advantage with CII was only seen among patients who remained in the ICU and continued to receive CII for 5 days or longer. This finding corroborates our assertion that 
strict glycometabolic correction and continuation of CII therapy for the day of surgery and at least the first 2 PODs are both key elements in the clinical success of our protocol.

Our study is the first to show a decrease in CABG mortality with insulin infusions. We believe that the previously published basic scientific literature supports our postulate that this mortality reduction has been brought about by enhanced glycometabolic control, with a resultant reduction in FFA intermediates. This nonsurgical intervention reduced both absolute and risk-adjusted mortalities in patients with DM undergoing CABG. The striking relationship of glucose levels to cardiac-related death also implicates a potential myocardial glycometabolic etiology for the improved survival. These findings corroborate our methodologic theory that myocardial energetics are enhanced by strict glycometabolic control.

\section{Summary}

Perioperative glycometabolic control with CII on the day of surgery and through the first 2 PODs reduced absolute mortality in our population of patients with DM undergoing CABG by $57 \%$. The reduction in mortality was completely accounted for by a reduction in cardiac-related deaths. Conversely overall mortality, specifically cardiac-related mortality, increased significantly in association with rising postoperative glucose levels. These findings implicate enhanced myocardial glycometabolic function as the underlying source of improved outcomes with CII. Strict glycometabolic control with CII normalized CABG mortality among patients with DM in our institution to that of the nondiabetic population. CII decreased risk-adjusted mortality by $50 \%$ and thus exerted a protective effect on mortality independent of the constellation of risk factors in the STS CABG risk model.

\section{Conclusion}

We conclude that DM per se is not a true risk factor for death after CABG. Rather, we propose that it is the underlying glycometabolic state of the myocardium that independently affects postoperative mortality. Excellent glycometabolic control can be safely achieved through the use of a CII in the perioperative period. Insulin infusions may induce biochemical changes in the production of myocardial ATP that are beneficial to cellular integrity and endothelial and ventricular function. This is amplified clinically into reduced postoperative mortality. Insulin infusions in patients with DM undergoing CABG reduce mortality and eliminate the incremental increase in risk-adjusted mortality previously ascribed to DM. Insulin infusions should become the standard of care for glycometabolic control in patients with DM undergoing CABG.

\section{References}

1. Edwards FH, Grover FL, Shroyer AL, Schwartz M, Bero J. The Society of Thoracic Surgeons National Cardiac Surgery Database: current risk assessment. Ann Thorac Surg. 1997;63:903-8.

2. Shroyer AL, Plomondon ME, Grover FL, Edwards FH. The 1996 coronary artery bypass risk model: the Society of Thoracic Surgeons Adult Cardiac National Database. Ann Thorac Surg. 1999;67:1205-8.

3. Thourani VH, Weintraub WS, Stein B, Gebhart SS, Craver JM, Jones EL, et al. Influence of diabetes mellitus on early and late outcome after coronary artery bypass grafting. Ann Thorac Surg. 1999;67:1045-52.

4. Harris MI. Classification and diagnostic criteria for diabetes mellitus and other categories of glucose intolerance. Prim Care. 1988;15:20525.

5. Stephens JW, Krause AH, Peterson CA, Bass JJ, Hartman JE, Salomon NW, et al. The effect of glucose priming solutions in diabetic patients undergoing coronary artery bypass grafting. Ann Thorac Surg. 1988;45:544-7.

6. Salomon NW, Page US, Okies JE, Stephens J, Krause AH, Bigelow JC. Diabetes mellitus and coronary artery bypass. Short-term risk and long-term prognosis. J Thorac Cardiovasc Surg. 1983;85:264-71.

7. Malmberg K, Ryden L, Hamsten A, Herlitz J, Waldenstrom A, Wedel H. Effects of insulin treatment on cause-specific one-year mortality and morbidity in diabetic patients with acute myocardial infarction. DIGAMI Study Group. Diabetes Insulin-Glucose in Acute Myocardial Infarction. Eur Heart J. 1996;17:1337-44.

8. Malmberg K, Norhammar A, Wedel H, Ryden L. Glycometabolic state at admission: important risk marker of mortality in conventionally treated patients with diabetes mellitus and acute myocardial infarction: long-term results from the Diabetes and Insulin-Glucose Infusion in Acute Myocardial Infarction (DIGAMI) study. Circulation 1999;99:2626-32.

9. Diaz R, Paolasso EA, Piegas LS, Tajer CD, Moreno MG, Corvalan R, et al. Metabolic modulation of acute myocardial infarction. The ECLA (Estudios Cardiologicos Latinoamerica) Collaborative Group. Circulation. 1998;98:2227-34.

10. Lazar HL, Philippides G, Fitzgerald C, Lancaster D, Shemin RJ, Apstein C. Glucose-insulin-potassium solutions enhance recovery after urgent coronary artery bypass grafting. J Thorac Cardiovasc Surg. 1997;113:354-60.

11. Rao V, Borger MA, Weisel RD, Ivanov J, Christakis GT, Cohen G, et al. Insulin cardioplegia for elective coronary bypass surgery. J Thorac Cardiovasc Surg. 2000;119:1176-84.

12. Lazar HL, Chipkin S, Philippides G, Bao Y, Apstein C. Glucoseinsulin-potassium solutions improve outcomes in diabetics who have coronary artery operations. Ann Thorac Surg. 2000;70:145-50.

13. Randle PJ. Metabolic fuel selection: general integration at the wholebody level. Proc Nutr Soc. 1995;54:317-27.

14. Furnary AP, Zerr KJ, Grunkemeier GL, Heller CA. Hyperglycemia: a predictor of mortality following CABG in diabetics. Circulation. 1999(Suppl);100:I591.

15. O'Connor GT, Birkmeyer JD, Dacey LJ, Quinton HB, Marrin CA, Birkmeyer NJ, et al. Results of a regional study of modes of death associated with coronary artery bypass grafting. Northern New England Cardiovascular Disease Study Group. Ann Thorac Surg. 1998; 66:1323-8.

16. Zerr KJ, Furnary AP, Grunkemeier GL, Bookin S, Kanhere V, Starr A. Glucose control lowers the risk of wound infection in diabetics after open heart operations. Ann Thorac Surg. 1997;63:356-61.

17. Hosmer DW, Lemeshow S. Applied logistic regression. New York: John Wiley and Sons; 2000.

18. Furnary AP, Zerr KJ, Grunkemeier GL, Starr A. Continuous intravenous insulin infusion reduces the incidence of deep sternal wound infection in diabetic patients after cardiac surgical procedures. Ann Thorac Surg. 1999;67:352-60.

19. Benson K, Hartz AJ. A comparison of observational studies and randomized, controlled trials. N Engl J Med. 2000;342:1878-86.

20. Concato J, Shah N, Horwitz RI. Randomized, controlled trials, observational studies, and the hierarchy of research designs. N Engl J Med. 2000;342:1887-92.

21. Taegtmeyer H. Carbohydrate interconversions and energy production. Circulation. 1985;72(5 Pt 2):IV1-8. 
22. Saddik M, Lopaschuk GD. Myocardial triglyceride turnover and contribution to energy substrate utilization in isolated working rat hearts. J Biol Chem. 1991;266:8162-70.

23. Saddik M, Gamble J, Witters LA, Lopaschuk GD. Acetyl-CoA carboxylase regulation of fatty acid oxidation in the heart. J Biol Chem. 1993;268:25836-45.

24. Kantor PF, Dyck JR, Lopaschuk GD. Fatty acid oxidation in the reperfused ischemic heart. Am J Med Sci. 1999;318:3-14.

25. Hasin Y, Barry WH. Myocardial metabolic inhibition and membrane potential, contraction, and potassium uptake. Am J Physiol. 1984; 247(2 Pt 2):H322-9.

26. Meyer C, Schwaiger M. Myocardial blood flow and glucose metabolism in diabetes mellitus. Am J Cardiol. 1997;80(3A):94A-101A.

27. Ferrannini E, Santoro D, Bonadonna R, Natali A, Parodi O, Camici PG. Metabolic and hemodynamic effects of insulin on human hearts. Am J Physiol. 1993;264:E308-15.

28. Oliver MF, Opie LH. Effects of glucose and fatty acids on myocardial ischaemia and arrhythmias. Lancet. 1994;343:155-8.

29. Mjos OD. Effect of free fatty acids on myocardial function and oxygen consumption in intact dogs. J Clin Invest. 1971;50:1386-9.

30. Rao V, Merante F, Weisel RD, Shirai T, Ikonomidis JS, Cohen G, et al. Insulin stimulates pyruvate dehydrogenase and protects human ventricular cardiomyocytes from simulated ischemia. $J$ Thorac Cardiovasc Surg. 1998;116:485-94.

31. Lazar HL. Enhanced preservation of acutely ischemic myocardium and improved clinical outcomes using glucose-insulin-potassium (GIK) solutions. Am J Cardiol. 1997;80(3A):90A-93A.

32. Lazar HL. Enhanced preservation of acutely ischemic myocardium using glucose-insulin-potassium solutions. J Card Surg. 1994;9 Suppl: 474-8.

33. Liedtke AJ. Lipid burden in ischemic myocardium. J Mol Cell Cardiol. 1988;20 Suppl 2:65-74.

34. Hess ML, Okabe E, Poland J, Warner M, Stewart JR, Greenfield LJ. Glucose, insulin, potassium protection during the course of hypothermic global ischemia and reperfusion: a new proposed mechanism by the scavenging of free radicals. J Cardiovasc Pharmacol. 1983;5:3543.

35. Knuuti MJ, Maki M, Yki-Jarvinen H, Voipio-Pulkki LM, Harkonen R, Haaparanta M, et al. The effect of insulin and FFA on myocardial glucose uptake. J Mol Cell Cardiol. 1995;27:1359-67.

36. Fietsam R Jr, Bassett J, Glover JL. Complications of coronary artery surgery in diabetic patients. Am Surg. 1991;57:551-7.

37. Rao V, Ivanov J, Weisel RD, Cohen G, Borger MA, Mickle DA. Lactate release during reperfusion predicts low cardiac output syndrome after coronary bypass surgery. Ann Thorac Surg. 2001;71: 1925-30.

38. Smedira NG, Blackstone EH. Postcardiotomy mechanical support: risk factors and outcomes. Ann Thorac Surg. 2001;71 Suppl:S60-6.

39. Whitlow PL, Rogers WJ, Smith LR, McDaniel HG, Papapietro SE, Mantle JA, et al. Enhancement of left ventricular function by glucoseinsulin-potassium infusion in acute myocardial infarction. Am J Cardiol. 1982;49:811-20.

40. Lazar HL, Zhang X, Rivers S, Bernard S, Shemin RJ. Limiting ischemic myocardial damage using glucose-insulin-potassium solutions. Ann Thorac Surg. 1995;60:411-6.

41. Ramanathan T, Shirota K, Morita S, Nishimura T, Huang Y, Hunyor SN. Glucose-insulin-potassium solution improves left ventricular mechanics in diabetes. Ann Thorac Surg. 2002;73:582-7.

42. van den Berghe G, Wouters P, Weekers F, Verwaest C, Bruyninckx F, Schetz M, et al. Intensive insulin therapy in the critically ill patients. N Engl J Med. 2001;345:1359-67.

\section{Appendix}

\section{Portland CII Protocol (Version 2001)}

Target blood glucose is 100 to $150 \mathrm{mg} / \mathrm{dL}$.

1. Start Portland protocol during surgery and continue through 7 AM of the third POD. Patients who are not receiving enteral nutrition on the third POD should remain on this protocol
Appendix TABLE 1. Parameters for CII under Portland protocol

\begin{tabular}{|c|c|c|c|}
\hline \multirow[b]{2}{*}{$\begin{array}{l}\text { Blood glucose } \\
\text { (mg/dL) }\end{array}$} & \multirow{2}{*}{$\begin{array}{l}\text { Intravenous } \\
\text { insulin bolus } \\
\text { (units) }\end{array}$} & \multicolumn{2}{|c|}{ Initial insulin rate $(\mathrm{U} / \mathrm{h})$} \\
\hline & & $\begin{array}{c}\text { Non-insulin } \\
\text { dependent DM }\end{array}$ & $\begin{array}{c}\text { Insulin } \\
\text { dependent DM }\end{array}$ \\
\hline $80-119$ & 0 & 0.5 & 1 \\
\hline $120-179$ & 0 & 1 & 2 \\
\hline $180-239$ & 0 & 2 & 3.5 \\
\hline $240-299$ & 4 & 3.5 & 5 \\
\hline $300-359$ & 8 & 5 & 6.5 \\
\hline$\geq 360$ & 12 & 6.5 & 8 \\
\hline
\end{tabular}

until receiving at least $50 \%$ of a full liquid or soft American Diabetes Association diet.

2. For patients with previously undiagnosed DM who have hyperglycemia, start Portland protocol if blood glucose is greater than $200 \mathrm{mg} / \mathrm{dL}$. Consult endocrinologist on POD 2 for DM workup and follow-up orders.

3. Start infusion by pump piggyback to maintenance intravenous line as shown in Appendix Table 1.

4. Test blood glucose level by finger stick method or arterial line drop sample. Frequency of blood glucose testing is as follows:

a. When blood glucose level greater than $200 \mathrm{mg} / \mathrm{dL}$, check every 30 minutes.

b. When blood glucose level is less than $200 \mathrm{mg} / \mathrm{dL}$, check every hour.

c. When titrating vasopressors, (eg, epinephrine) check every 30 minutes.

$\mathrm{d}$. When blood glucose level is 100 to $150 \mathrm{mg} / \mathrm{dL}$ with less than $15 \mathrm{mg} / \mathrm{dL}$ change and insulin rate remains unchanged for 4 hours ("stable infusion rate"), then you may test every 2 hours.

e. You may stop testing every 2 hours on POD 3 (see items 1 and 8$)$.

f. At night on telemetry unit, test every 2 hours if blood glucose level is 150 to $200 \mathrm{mg} / \mathrm{dL}$; test every 4 hours if blood glucose level is less than $150 \mathrm{mg} / \mathrm{dL}$ and "stable infusion rate" exists.

5. Insulin titration according to blood glucose level is performed as follows

a. When blood glucose level is less than $50 \mathrm{mg} / \mathrm{dL}$, stop insulin and give $25 \mathrm{~mL} \mathrm{50 \%} \mathrm{dextrose} \mathrm{in} \mathrm{water.} \mathrm{Recheck}$ blood glucose level in 30 minutes. When blood glucose level is greater than $75 \mathrm{mg} / \mathrm{dL}$, restart with rate $50 \%$ of previous rate.

b. When blood glucose level is 50 to $75 \mathrm{mg} / \mathrm{dL}$, stop insulin. Recheck blood glucose level in 30 minutes; if previous blood glucose level was greater than 100 then give $25 \mathrm{~mL}$ $50 \%$ dextrose in water. When blood glucose level is greater than $75 \mathrm{mg} / \mathrm{dL}$, restart with rate $50 \%$ of previous rate.

c. When blood glucose level is 75 to $100 \mathrm{mg} / \mathrm{dL}$ and less than $10 \mathrm{mg} / \mathrm{dL}$ lower than last test, decrease rate by $0.5 \mathrm{U} / \mathrm{h}$. If blood glucose level is more than $10 \mathrm{mg} / \mathrm{dL}$ lower than last 
test, decrease rate by $50 \%$. If blood glucose level is the same or greater than last test, maintain same rate.

d. When blood glucose level is 101 to $150 \mathrm{mg} / \mathrm{dL}$, maintain rate.

e. When blood glucose level is 151 to $200 \mathrm{mg} / \mathrm{dL}$ and 20 $\mathrm{mg} / \mathrm{dL}$ lower than last test, maintain rate. Otherwise increase rate by $0.5 \mathrm{U} / \mathrm{h}$.

j. When blood glucose level is greater than $200 \mathrm{mg} / \mathrm{dL}$ and at least $30 \mathrm{mg} / \mathrm{dL}$ lower than last test, maintain rate. If blood glucose level is less than $30 \mathrm{mg} / \mathrm{dL}$ lower than last test (or is higher than last test), increase rate by $1 \mathrm{U} / \mathrm{h}$ and, if greater than $240 \mathrm{mg} / \mathrm{dL}$, administer intravenous bolus of regular insulin per initial intravenous insulin bolus dosage scale (see item 3). Recheck blood glucose level in 30 minutes.

$\mathrm{k}$. If blood glucose level is greater than $200 \mathrm{mg} / \mathrm{dL}$ and has not decreased after three consecutive increases in insulin, then double insulin rate.

b. If blood glucose level is greater than $300 \mathrm{mg} / \mathrm{dL}$ for four consecutive readings, call physician for additional intravenous bolus orders.

6. American Diabetes Association 1800-kcal diabetic diet starts with any intake by mouth.

7. Postmeal subcutaneous Humalog insulin supplement is given in addition to insulin infusion when oral intake has advanced beyond clear liquids.

a. If patient eats $50 \%$ or less of servings on breakfast, lunch, or dinner tray, then give 3 units of Humalog insulin subcutaneously immediately after that meal.

b. If patient eats more than $50 \%$ of servings on breakfast, lunch, or supper tray, then give 6 units of Humalog insulin subcutaneously immediately after that meal.

8. On third POD, restart preadmission glycemic control medication unless patient is not tolerating enteral nutrition and is still receiving an insulin drip.

\section{Discussion}

Dr Terrence M. Yau (Toronto, Ontario, Canada). I congratulate Furnary and colleagues on identifying a subgroup of patients undergoing $\mathrm{CABG}$ who are clearly at higher risk for perioperative morbidity and mortality and for devising a physiologically based, if clinically unproven, method to improve clinical outcomes.

Interest in metabolic enhancement of the ischemic myocardium started as early as 1965, when Sodi-Pollares observed the beneficial effects of a continuous GIK infusion on the electrocardiographic changes after acute MI. Early excitement about this form of therapy, both as a continuous intravenous infusion and as a bolus cardioplegic additive, waned as several small randomized trials in a variety of cardiovascular settings failed to demonstrate a consistently protective effect. Caution was then introduced by David Hearse in 1978, when he documented an exacerbation of ischemic injury in rats rendered hyperglycemic during the period of cardioplegic arrest.

Recently our group in Toronto and Lazar's group in Boston have revisited the concept of perioperative metabolic enhancement with GIK solutions. In addition, the Portland group of Furnary and colleagues have performed an extensive series of investigations into the effects of insulin on clinical outcomes in patients with
DM, including this analysis. However, one must exercise caution before interpreting the results of any clinical study, particularly a nonrandomized retrospective observational review.

Furnary and colleagues are to be commended for a thorough statistical analysis of their data, but as they indicate in their article, no amount of statistical adjustment can adequately replace the robust design of a prospective, randomized study. Any post hoc statistical adjustment is only as good as the variables that are entered into that model. One approach to remove this bias is to perform a propensity analysis and then adjust your findings according to the likelihood of a given patient with DM receiving either SQI or CII.

I have a few questions for Dr Furnary. Your institution favors the use of intermittent fibrillatory arrest for perioperative myocardial protection. Do you believe that your observed differences would still be significant if your surgeons used blood cardioplegia? In addition, it appears from your data that the primary benefit of CII was a reduction in cardiac-related mortality relative to patients receiving SQI. However, there was no difference in the postoperative use of inotropes or in postoperative low cardiac output syndrome in these patients. You do not mention the prevalence of perioperative MI. Was your observed benefit due to reduction in postoperative arrhythmias, as Lazar has previously shown, or truly an improvement in myocardial function, as you imply in your article?

You comment that your institutional review board would no longer consider it ethical to perform a prospective randomized trial on CII in patients with DM. Are you considering such a trial in patients without DM where you could randomly assign them to receive CII with tight glucose control versus placebo? You may wish to design such a trial to specifically examine the prevalence of postoperative arrhythmias.

Finally, although I personally favor the use of perioperative insulin, I must report to the audience that a large multicenter prospective clinical trial, to be published shortly in our Association's journal, failed to demonstrate a clinical benefit of insulinenhanced cardioplegia in patients undergoing urgent CABG. We do believe, however, that perhaps the true benefit of insulin infusion may be seen in patients with moderate to severe left ventricular dysfunction, and we are currently enrolling patients in a randomized evaluation of that intervention.

A substudy of our trial on patients undergoing urgent revascularization failed to demonstrate any reduction in the postoperative incidence of supraventricular tachyarrhythmias. These trials have used insulin only as a cardioplegic additive and failed to administer the drug beyond the operating room. It is conceivable that following a protocol similar to that described today by the Portland group might result in clinically meaningful benefit. I believe that such a prospective randomized study is worthy of consideration.

Dr Furnary. I will take your questions in order. We do use intermittent fibrillatory arrest as a method of myocardial protection. The periods of arrest range between 5 and 12 minutes, after which the heart is reperfused. We have not seen a difference in mortality in our CABG population without DM and that predicted by the STS risk model. As a matter of fact, our observed/expected mortality ratio in that population is consistently in the 0.5 to 0.7 range. So I do not believe that mortality reduction from insulin infusion is unique to its combination with intermittent fibrillation. 
I believe that the mortality benefits are seen along with the use of cardioplegia as well.

Backing up that statement is the fact that this study focused only on patients undergoing isolated CABG. When we looked at the excluded subset of 400 additional patients who underwent combined CABG and valve replacement and who had blood cardioplegia delivery as a method of myocardial protection, almost the exact same multiple logistic regression came up, with insulin being protective against postoperative death.

In answer to your second question, we actually do know the incidences of low cardiac output and arrhythmia. We do not know the incidence of postoperative MI. I would point out that although this was a nonrandomized study, it was a prospective interventional study (not a retrospective observational one) in which we collected our data in a prospective fashion.

The incidence of low cardiac output in the entire population was $11 \%$. The graph of the relationship of low cardiac output to increase in glucose levels exactly mirrors the graph of increasing mortality with increasing glucose levels. Therefore I believe that ventricular function is a key component in the improved survival seen with insulin infusions, which strictly control glucose levels. In addition, arrhythmias are much more prevalent with high glucose levels. And when we examine the biochemical energetics of the myocardial cell and look at what is happening at the cellular level, it is easy to see why this would be so. As a matter of fact, in Lazar's published work with GIK in patients with DM, he also noted a decreased incidence of arrhythmias, including atrial arrhythmias such as atrial fibrillation.

In terms of a randomized trial, at this point we certainly could not ethically perform a randomized trial in our institution comparing CII and SQI. We have considered doing a randomized trial comparing extremely low glucose levels, perhaps a target range of 80 to $110 \mathrm{mg} / \mathrm{dL}$, with our current target range of 100 to 150 $\mathrm{mg} / \mathrm{dL}$.

As you can see, our protocol has been modified with time. The goal at the outset of this project was to gradually and safely establish euglycemia in all patients with DM in an attempt to modify outcomes and normalize those outcomes to those of the population without DM. So theoretically we could randomly assign one group of patients to a target range between 80 and 120 $\mathrm{mg} / \mathrm{dL}$ and another group to a target range between 150 and 200 $\mathrm{mg} / \mathrm{dL}$. The problem comes in that the power analysis tells us that to show a $50 \%$ decrease in mortality between those two groups, we are going to need around 5000 patients, and we only accumulate about 200 patients with DM undergoing CABG per year. Thus such as study would have to be done in a multi-institutional setting.

Finally, the fact that insulin-enhanced cardioplegia shows no overall clinical benefits in your upcoming study is not surprising to me. It reinforces the fact that insulin therapy must be continued for some time after the operation to show a clinical effect on mortality. In Rao's previous article on insulin-enhanced cardioplegia, there was no benefit to ventricular function seen beyond 8 hours after the operation in patients without DM. And one must further understand that patients with $\mathrm{DM}$ undergoing $\mathrm{CABG}$ not only have the detriment of ischemia of the myocardium but also the ongoing, persistent detriment of the lack of effective oxidative glycolysis. Those two things combined are what make the patient with DM unique, not only in surgery but in the postoperative period as well.
And that is why we believe, as was published in the New England Journal of Medicine this past November, that the prolonged use of insulin and thus prolonged normoglycemia are important in improving clinical outcomes.

Dr Richard M. Engelman (Springfield, Mass). I congratulate Furnary and colleagues on a sophisticated presentation and on bringing this subject, which has important implications for all of us in the care of the patient with DM, to our attention. I have had an opportunity to review the manuscript and thus can speak from some data.

One point that I would like to emphasize is the potential importance of intraoperative glycemia control. This was not actually controlled, as pointed out by Furnary and colleagues, until 1996, and the absence of rigorous control of the intraoperative blood sugar may be a factor in the results reported here. Intraoperative hyperglycemia in most hospitals is treated by the anesthesiologist without surgical involvement or adherence to a protocol. I have the impression that intraoperative hyperglycemia is a factor in leading to depressed myocardial recovery. The most frequent cause of death in the series, as the article points out, was cardiac related. Dr Furnary, I would appreciate your comments on that.

A question that I have relates to the actual solution that you used for CII. We are about to enter a protocol for a glycemic control with a glucose-insulin solution, and I would be interested in knowing what your solution was and whether you believe glucose added to the insulin has any advantage in preventing hypoglycemia or simply makes glycemic control more difficult.

My final question relates to optimal glycemic level. You have now reduced your blood sugar goal to $120 \mathrm{mg} / \mathrm{dL}$, as you just indicated, from a high of $200 \mathrm{mg} / \mathrm{dL}$ initially, and the recently published Belgium study, as you mentioned in the New England Journal of Medicine, actually used a goal of 80 to $110 \mathrm{mg} / \mathrm{dL}$. As you have shown no difference in mortality related to the year of this study once the patients were receiving the CII, why do you believe that a lower glycemic level is actually preferable, or is less than $200 \mathrm{mg} / \mathrm{dL}$ actually satisfactory?

Dr Furnary. First of all, I wholeheartedly agree with your assertion that intraoperative hyperglycemia is paramount in affecting outcomes, especially cardiac-related outcomes. At the current time we manage intraoperative hyperglycemia along with the anesthesiologists and actually write the numbers up on the board so that everybody can see what they are. It was somewhat difficult to get that started, because some anesthesiologists preferred just to give bolus insulin and preferred not to institute a drip. But after we got it going, it really had a marked effect on postoperative glucose control and hence clinical outcomes, and I could not agree more with your statement.

The solution that we currently use is 125 units of regular insulin in $250 \mathrm{~mL}$ isotonic saline solution. The reason that we use isotonic saline solution is we use it as a piggyback infusion along with another intravenous drip. When we were using 5\% dextrose in water solutions as a maintenance intravenous solution, some patients without DM would become hyperglycemic because of the combined stresses of surgery in the face of added glucose. As glucose levels rose in these patients without DM, they would get started on an insulin drip. When they were transferred to the floor and as they started eating, the 5\% dextrose in water maintenance infusions were discontinued; however, the insulin infusions con- 
tinued to run. These patients without DM would thus end up with hypoglycemia as they started eating and the 5\% dextrose in water intravenous drip was stopped, and that was not good. To avoid that situation, we switched to isotonic saline solution as our standard intravenous solution. We thus decreased the incidence of iatrogenic hyperglycemia in patients without DM and also decreased the incidence of hypoglycemia on the telemetry floor.

The optimal glucose level for enhancing clinical outcomes is uncertain. I can tell you that if you keep it below $150 \mathrm{mg} / \mathrm{dL}$, you will certainly see the effects on wound infection rates that we have previously demonstrated and the reductive effect on mortality demonstrated today. The intent of lowering it to $120 \mathrm{mg} / \mathrm{dL}$ is to complete our original intent of this study, which was to normalize glucose levels in these patients and thus try to normalize heart surgery outcomes of patients with DM to those of the general population. What I believe lowering the target levels may do is to lower the incidence of atrial fibrillation, and we are already seeing a trend in that direction.

But the two key elements here for anyone putting this type of protocol together are glucose target levels and duration of the infusion. Glucose levels should be tightly controlled at near normoglycemia or euglycemia, and the insulin should be used for a period of at least 3 days (the day of surgery and the first and second PODs) to effect the outcomes that we have described. And that rationale has to do, as explained in the article, with the mechanistic effects of glycolysis-derived cytosolic ATP on membrane function and integrity in the postoperative period.

Dr Harold I. Lazar (Boston, Mass). Three years ago, we had the privilege of presenting some data on patients with DM. We used a modified GIK solution, and we did find significant improvement, with reduction in atrial fibrillation, weight gain, improved cardiac index, and decreased length of stay.

My comments are related to the mechanism responsible for the beneficial effects of GIK in these patients. The mechanism may not necessarily be due to the increased availability of ATP. In the last 2 years we have had the opportunity to continue this study and actually measure not only the level of glucose, which we have maintained between 120 and $180 \mathrm{mg} / \mathrm{dL}$, but also FFAs, triglycerides, and lactate. We found that there is a beneficial effect of GIK in decreasing FFAs. These beneficial effects may not all be related to increased availability of ATP but rather to improvement in endothelial function.

My second point is when you give this solution. It is important to start it in the operating room during induction and continue it for at least 12 to 24 hours. This explains why Dr Yau's insulin cardioplegia had very little effect. It was only given for a short period. Clearly, this metabolic enhancement has to be given for a longer period.

With respect to the other comment about doing a study in patients without DM, as you know, we have already done that. Certainly insulin and glucose together in a population without DM are also extremely effective, especially in patients with unstable and emergency CABG surgery.
I think that it is not ethical to randomly assign these patients anymore, because tighter glycemic control for these patients leads to better clinical outcomes. We have found that using 5\% dextrose in water and insulin in a drip has no detrimental effect. So these people can have a little bit of glucose. In fact, one of the studies that we are currently doing in patients with DM is to see whether there is any advantage in adding glucose to insulin, versus just an insulin drip alone.

Dr Furnary. Dr Lazar, it is a privilege to have you comment on this study. I could not agree with you more that there is more going on than just intracellular ATP modification, and we go into many of the other probable mechanisms in the article. In an 8 -minute presentation it is difficult to cover all the possible biochemical mechanisms. But the elimination of the toxic intermediates of FFA oxidation no question plays a key role in eliminating the no-reflow phenomenon, improving endothelial function, and stabilizing cellular membranes.

However, I would take that comment and go into your second question, and this I think is where we disagree. It is my firm belief that insulin infusions should be used not just for 12 hours after the operation, as in your study, but for longer than 48 hours. Thus, for consistency in this regard, we discontinue our insulin infusion protocol on the morning of the third POD. The rationale for that duration is that, as we all know from our basic surgical training, the first 48 postoperative hours is the period of maximal edema accumulation. Decreased FFA intermediates and increased cytosolic ATP are extremely important in stabilizing membrane function. Thus to achieve the maximum benefits on those parameters that you have shown in your articles to be potentially influenced by insulin metabolic modification-decreased edema, decreased intubation times, and decreased weight gain-one must use the protocol through the first 3 PODs. That is the duration through which we believe this protocol must be used. I do not think that the same mortality-reducing results would be seen if the protocol were used for a shorter time than 3 PODs.

Finally, with regard to whether random assignment is ethical, we can look at it this way. We in medicine hold ourselves to a high standard so as not to harm our patients while obtaining definitive answers to our scientific questions. Because of this, we prefer to use randomized controls. But this study and this protocol was a process of continuous quality improvement with known and ongoing outcome improvements in infection control and length of stay; to randomize the protocol at this point would be like having the automobile industry randomize the placement of seat belts in the late 1970s. Think of the countless people who would have died in that process before that industry said, "Oh, seat belts work, let's put them in every car." So I think that we, especially today with the social and economic backgrounds against which we currently work in medicine, have to do studies and make decisions that make pragmatic sense for our patients and their lives, while still deriving what scientific data we can from those studies in a sensible way. 